\title{
Siew-Ging Gong \\ Characterization of olfactory nerve abnormalities in Twirler mice
}

Accepted in revised form: 20 August 2001

\begin{abstract}
The sense of smell is perceived by olfactory receptor neurons $(\mathrm{ORN})$ present in the olfactory epithelium located in the posterosuperior aspect of the nasal cavity. The axons of these ORN migrate to the olfactory bulb (OB), forming a nervous layer on the outermost part of the bulb, and finally synapse in glomerular structures in the OB. The ORN are unique in that they are constantly being renewed throughout life. We characterized the defects in the nasal cavity and olfactory nervous supply of Twirler (Tw) mice by histological and immunohistochemical means. Tw homozygotes have previously been shown to present with midfacial abnormalities in the form of clefts of the lip and palate (Lyon, 1958; Gong et al., 2000). We found that in the Tw homozygotes, the OB was abnormally shaped, the skeletal framework underlying the $\mathrm{OB}$ was disrupted, and the morphology of the nasal cavity was altered with poorly defined nasal turbinates. Immunohistochemical staining with antibodies that marked nerves in general (PGP 9.5) and mature ORN (omp) in the olfactory epithelium at two different embryonic stages and in newborn mice revealed the stratification of the olfactory epithelium in $T w$ homozygotes, albeit slightly thinner compared to wildtype. A striking difference in the olfactory epithelium was the lack of differentiation of the ORN in $T w$ homozygotes and the reduced axonal input to the OB. In $T w$ homozygotes at 14.5 days of embryonic development, the presence of many mature ORN found randomly in the mesenchyme suggests the loss of olfactory
\end{abstract}

\section{S.-G. Gong}

Department of Orthodontics and Pediatric Dentistry

School of Dentistry

University of Michigan

1011 N. University Avenue

Ann Arbor, MI 48109-1078, USA

e-mail:sggong@umich.edu

Tel: +1 734763 5391, Fax: + 17347638100 pathfinding cues to the OB. It is believed that the lack of appropriate pathfinding cues observed in the $T w$ homozygotes was responsible for the $\mathrm{OB}$ not having the appropriate trophic effect on the development and maturation of the ORN as had been observed in partially bulbectomized animals. The defects in the Twirler may prove to be a valuable system to analyze problems in olfactory pathfinding and maturation.

Key words olfactory receptor neurons - olfactory bulb . clefts of the lip and palate $\cdot$ mouse model

\section{Introduction}

The first neurons in the vertebrate olfactory pathway are sensory cells located in the olfactory epithelium (OE) in the posterosuperior aspect of the nasal cavity. The olfactory epithelium is a pseudostratified columnar mucosa with zones characterized by the location of the nuclei of the various OE cell types, the olfactory receptor neuron (ORN), the sustentacular cells, and the basal cells, overlying a lamina propria. Olfactory receptor cells (ORN) are bipolar neurons with cell bodies in the olfactory epithelium and are unique in that they are constantly undergoing turnover even through adulthood (Moulton et al., 1970; Graziadei and Metcalf, 1971; Graziadei and Monti-Graziadei, 1978). Globose basal cells, located at the base of the neuroepithelium, are the self-renewing source of new ORN (Moulton et al., 1970; Graziadei and Metcalf, 1971; Graziadei, 1973; Moulton, 1974; Graziadei and Monti-Graziadei, 1979; Caggiano et al., 1994). The ORN synapse on the dendrites of mitral cells and juxtaglomerular interneurons in globular structures called glomeruli in the olfactory bulb (OB) (reviewed in Farbman, 1991).

The main OB develops as an outpocketing of the rostral end of the cerebral vesicles around the 12th day of 
embryonic development in mice. The OB initially comprises only two layers, and neuronal genesis occurs in the highly cellular ventricular layer. The growing axons of the olfactory nerves reach the OB soon after the bulb forms and remain in contact with bulbar cells for 2 days before actual synaptic specializations occur. Axons from the ORN form a layer on the outermost part of this telencephalic outpocketing, being separate yet apposed to it. The first synapses in mouse olfactory bulb are seen on the 14th embryonic day, between sensory axons and mitral cell dendrites in the presumptive glomerular layer of the OB. Beyond the 15th day of embryonic development, there are many more axodendritic synapses in the OB (reviewed in Farbman, 1991).

The sense of smell is perceived by receptors present in the cilia of the ORN. Evidence from numerous studies indicates that mammals have functional olfactory systems at birth or shortly after birth; these systems are capable of discriminating a wide range of odors (Shepherd, 1994; Buck, 1996). Olfaction drives basic patterns of behavior in animals such as the identification of food, enemies, and mating partners. ORN detect odorants as chemical stimuli, transduce and couple the information with the electrical properties of the neuron, and transmit this information to the brain with optimal accuracy (Kinnamon and Getchell, 1991). In humans, olfactory disturbance is common (Jones and Rog, 1998).

The Twirler model has been described as a system to analyze defects in midfacial growth (Gong et al., 2000; Gong and Eulenberg, in press). All $T w$ homozygotes have midfacial defects in the form of clefts of the palate and lip. Clefts of the palate in these mutants have a consistent shape and form, whereas clefts of the lip can be uni- or bilateral, and complete or incomplete. Preliminary observations indicated that there were also defects in the nasal cavity itself as well as in the development of the olfactory bulb. This paper is an analysis of the defects present in the olfactory system and the nasal cavity of the Twirler model.

\section{Methods}

Animals

The origin and maintenance of the $T w$ have been described (Lyon, 1958; Gong et al., 2000). To obtain timed pregnant embryos, two female mice were placed in the same cage with a male mouse overnight. The next morning, the presence of vaginal plugs in female mice was checked. If a vaginal plug was present, fertilization was assumed to occur at midnight and the embryos were considered to be 0.5 day in utero. The embryos were removed from euthanized female mice by caesarian section. Newborn pups were removed from their mothers within $12 \mathrm{~h}$ after birth. Genotyping of $T w$ mice and pups was performed according to Gong et al. (2000).

The total number of embryos utilized for this study were: newborns: 3 litters, with 4 homozygotes; $16.5 \mathrm{dpc}$ : 5 litters, with 6 homozygotes; and 14.5 dpc: 3 litters, with 5 homozygotes.
Tissue preparation

The craniofacial region of the mouse embryos and newborns were fixed in Bouin's fixative for a maximum of $24 \mathrm{~h}$, rinsed in $70 \%$ ethanol, and embedded in Paraplast. They were sectioned in different planes at $8 \mu \mathrm{m}$ thickness. Some of these sections were utilized for immunohistochemistry and some for histological analysis. For histology, regular hematoxylin and eosin stain was performed following standard protocols.

Immunohistochemical staining

Sections were first deparaffinized in xylene and hydrated in a series of alcohol washes $(100 \%, 95 \%$, and $70 \%)$. The sections were washed in phosphate-buffered saline (PBS) and normal blocking solution (10\% normal horse serum [NHS], $0.01 \%$ Saponin, $0.1 \%$ BSA in $1 \times$ PBS) was added. After excess serum was blotted from the sections, either anti-omp or -PGP 9.5 antibody (1:8000 dilution of goat anti-rat antibody against omp [gift of F. Margolis, University of Maryland] or 1:5000 dilution of rabbit anti-human polyclonal antibody against PGP 9.5 [Ultraclone, UK]) was added and left on overnight at $4{ }^{\circ} \mathrm{C}$. The next day, the slides were washed and the secondary antibody (for omp, $5 \%$ of biotinylated horse antigoat diluted in the $0.01 \%$ Saponin, $1.5 \%$ NHS, $1 \times$ PBS; for PGP $9.5,5 \%$ of biotinylated goat anti-rabbit) was added to the sections for $30 \mathrm{~min}$ at room temperature. After washing, the slides were quenched in $0.3 \%$ hydrogen peroxide in absolute methanol followed by amplification of the signal using Vectastain Elite ABC (Vector Labs, California) and detection with diaminobenzidine (DAB; $0.1 \%$ ). A standard 5-min incubation time in the DAB was used for all sections. After washing and dehydrating the slides in a series of $70 \%, 95 \%$, and $100 \% \mathrm{ETOH}$, they were mounted and analyzed.

\section{Results}

Architecture of the olfactory bulb (OB) and nasal cavity

Initial examination of the central nervous system (brain and spinal cord) revealed no apparent difference in size or gross organization among $T w$ homozygotes and the wildtype littermates; the only difference between these littermates was in the olfactory bulb (OB). Analysis of $\mathrm{OB}$ in newborn $T w / T w$ pups revealed their abnormal shape (Fig. 1B, white arrows). Instead of ending in a triangular cone at the rostral end of the cerebellar lobes (Fig. 1A), one and sometimes both bulbs extended into elongated tips (Fig. 1B, right bulb). The OB in the $T w /$ $T w$ frequently protruded into the nasal cavity through the ethmoid bone, readily observed in sagittal (Fig. 1D, black arrow) and frontal sections (Fig. 2D). This protrusion was accompanied by a breakage of the cribriform plate (Fig. 2, arrow in Fig. 2D). The presence of the unilateral cleft of the lip was evident (arrow in Fig. 2B) as observed in all $T w$ homozygotes (Gong et al., 2000).

The $T w$ mutation affected the shape and structure of the nasal cavity. In addition to clefts of the secondary palate observed in all $T w / T w$ mice (asterisks in Fig. 2D 


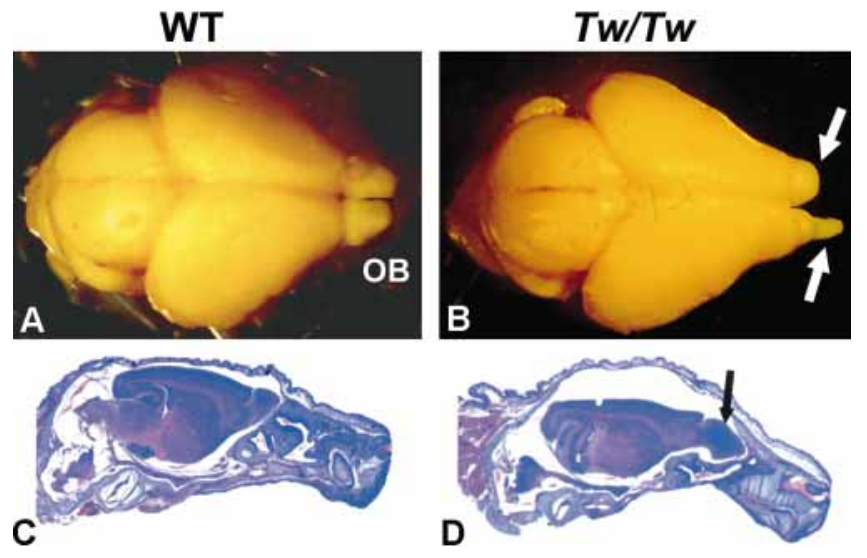

Fig. 1 Olfactory bulbs of newborn mice. The two olfactory bulbs $(O B)$ of wildtype newborn mice (A) present as two lobes rostral to the cerebellar lobes. In $T w / T w$ mice, the bulbs appeared irregular and asymmetrical in shape (arrows in B). In sagittal sections across the craniofacial region of wildtype $(\mathbf{C})$ and $T w / T w$ newborns, the $\mathrm{OB}$ protruded anteriorly into the nasal cavity (arrow in D).

and F, Fig. 4D; Gong et al., 2000; Gong and Eulenberg, in press), there were severe alterations in the morphology of the nasal cavity in these mice. Instead of the normal architecture of three distinct and well-defined superior, middle, and inferior turbinates of the nasal cavity in wildtype mice (Fig. 2A), the turbinates in $T w / T w$ were not clearly defined. The turbinates in $T w$ homozygotes were often present as ill-defined masses of tissues especially toward the posterior part of the nasal cavity. The disruption in the nasal cavity occurred concomitant with disruptions in the shape and development of the secondary palate previously described (Gong et al., 2000; Gong and Eulenberg, in press). The nasal septum in the mutant mice appeared more bulbous at the lower end where it should have fused with the secondary palate. The vomeronasal organ appeared normal and similar to those observed in normal littermates.

\section{Distribution of olfactory nerves in the $\mathrm{OB}$}

The disruption of the nasal cavity and $\mathrm{OB}$ in $T w / T w$ mice led to the suspicion that the olfactory nerves would be affected in these mice. In order to characterize the distribution of nerves in the craniofacial region of $T w /$ $T w$ mice, immunohistochemistry with the Protein Gene Product 9.5 (PGP 9.5) and olfactory marker protein (omp) antibodies was performed on sections through the craniofacial region of the $T w / T w$ at different stages of development. The polyclonal antibody against PGP 9.5 (Ultraclone Ltd, UK) will stain neuronal cell bodies and axons in the central and peripheral nervous system (Thompson et al., 1983; Wilson et al., 1988). The ability to synthesize a specific protein, the olfactory marker protein (OMP), is an important attribute of olfactory neurons (Margolis, 1972, 1975; Monti-Graziadei et al.,

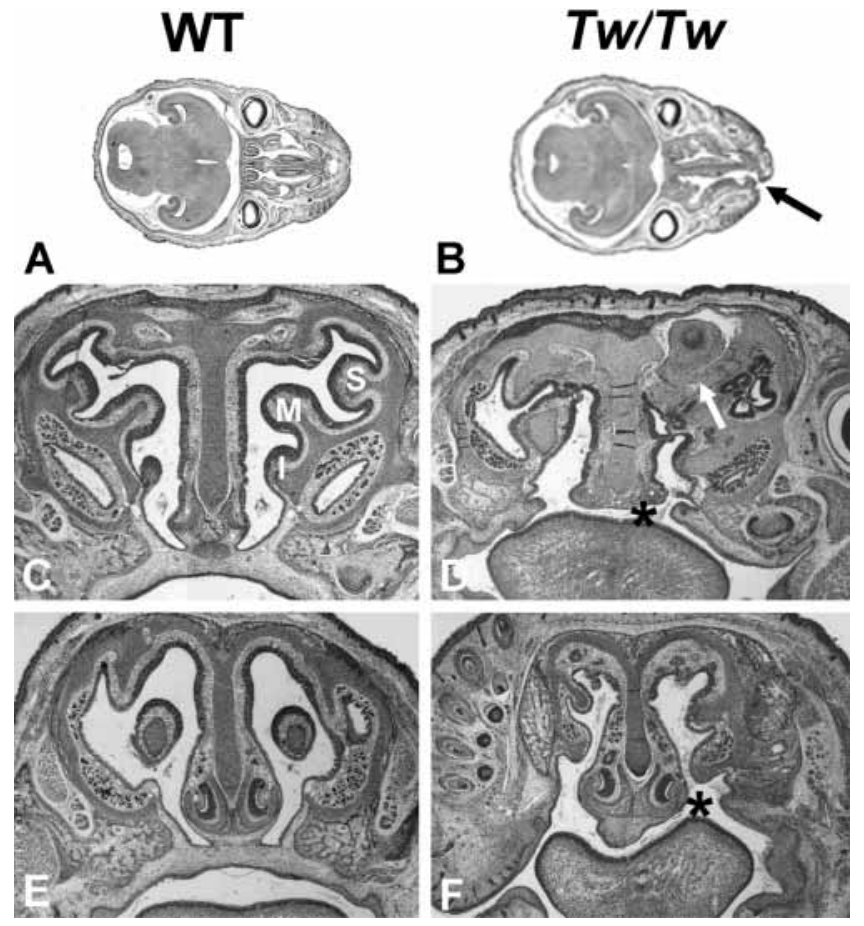

Fig. 2 Histological sections through the nasal cavity of wildtype and $T w / T w$ newborn mice. Horizontal sections through the level of the orbit in newborn wildtype (A) and $T w / T w$ (B) mice and coronal sections through the anterior third of the craniofacial region of wildtype (left panels) and Tw/Tw (right panels) mice. Note the presence of a cleft of the lip in $T w / T w$ mice (arrow in B). The general shape and structure of the nasal cavity in wildtype mouse looked relatively regular and symmetrical; the superior $(S)$, middle $(M)$, and inferior $(I)$ turbinates are well defined and regularly shaped (C). In the $T w / T w$ mice, the structure of the nasal cavity is irregularly shaped with no distinct turbinates and protrusion of left olfactory bulb through the bony roof of the nasal cavity (white arrow in D). In sections through two different frontal planes of the nasal cavity of wildtype mice (C and E) and $T w / T w$ mice (D and F), the irregularity of the shape and size of the nasal turbinates in $T w / T w$ was obvious especially in $\mathbf{D}$. The presence of the cleft of the secondary palate was also observed (asterisks in $\mathbf{B}$ and $\mathbf{F}$ ). The vomeronasal organ appeared similar in both wildtype and $T w / T w$ mice.

1977). OMP has been shown to be present only in the olfactory neuroepithelium and in the olfactory bulb of many vertebrates and is restricted to all parts of the differentiated ORN. OMP does not appear until embryonic day 14 in mouse. By day 16.5 there are significantly increased numbers of mature olfactory neurons, with an increased OMP staining.

In normal mice, afferent axons of ORN located in the olfactory epithelium penetrate the basal lamina of the olfactory epithelium in the nasal cavity and aggregate into fasciculi. These tracts of nerve bundles pass through foramina in the cribriform plate (black arrows in Figs $3 \mathrm{C}$ and $4 \mathrm{~A}$ ) immediately above the olfactory mucosa to terminate within the OB. The olfactory nerves enter the olfactory bulb to form the olfactory nerve layer in the olfactory bulb, the site of the first synaptic interactions 


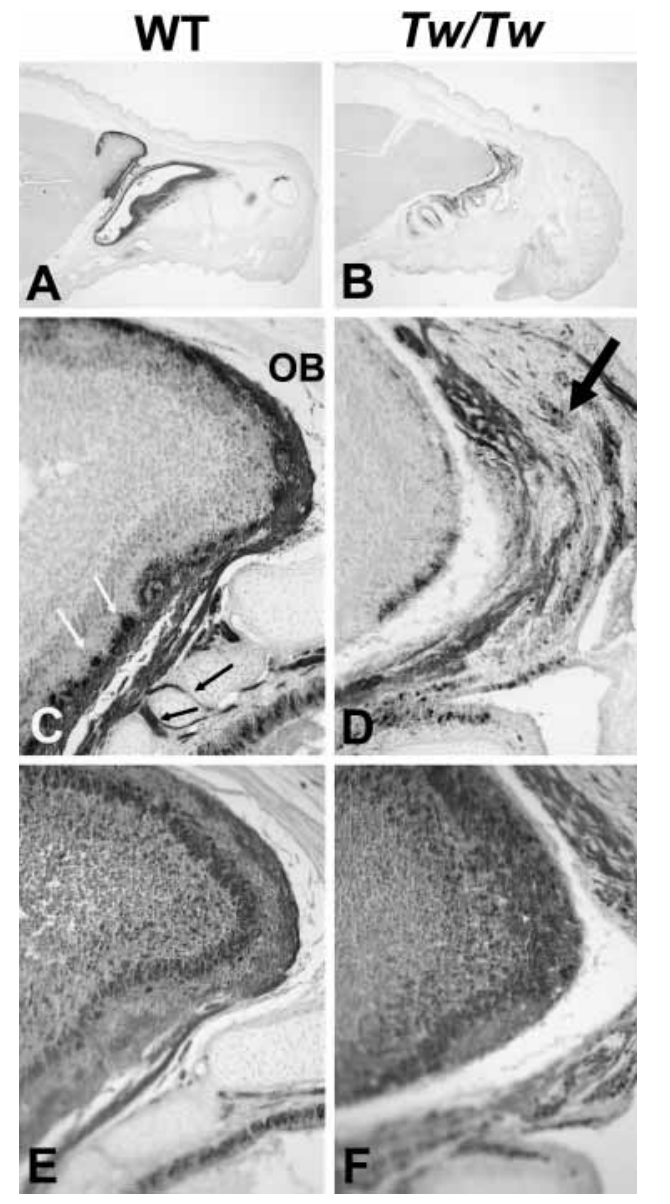

Fig. 3 Nervous supply in the olfactory bulb. Immunohistochemical staining with antibodies against olfactory marker protein, omp (AD), and PGP 9.5 (E and $\mathbf{F}$ ) on sagittal sections of wildtype (left panels) and $T w / T w$ (right panels). A, B: $4 \times$; C-F: $50 \times$ magnification. In wildtype mice, the outermost layer of the OB is covered with a layer of $\mathrm{omp}^{+}$cells, the axons of the olfactory neurons that terminate in glomerular structures (white arrows in $\mathbf{C}$ ). The olfactory epithelium in the nasal cavity contains ORN that are reactive to the omp antibody (A and $\mathbf{B})$. Tracts of the ORN pass through foramina in the cribriform plate of the ethmoid bone (black arrows in C). Unlike wildtype mice, there is no distinct layer of axons of olfactory neurons in the outermost layer of the OB. Instead, the axons are scattered randomly in the mesenchyme (arrow in $\mathbf{D})$. The OB appears as a layered structure due to the arrangement of different neuronal types in wildtype embryo $(\mathbf{E})$. The layered structure is not so apparent or ordered in $T w / T w$ mice (F).

in the olfactory pathway (Fig. 3C). The OB appeared as a layered structure due to the arrangement of different neuronal types in each layer (Fig. 3E). Many of these axons connect with the dendrites of mitral cells to form spherical structures called glomeruli (white arrows in Fig. 3C).

In the $T w / T w$, the olfactory nerves were not organized into distinct tracts or fasciculi. Instead, the nerves in these newborn mice appeared loosely packed and scattered in the mesenchyme of the nasal cavity (arrows in Fig. 3D and Fig. 4B) or present as clumps of fibers (ar-

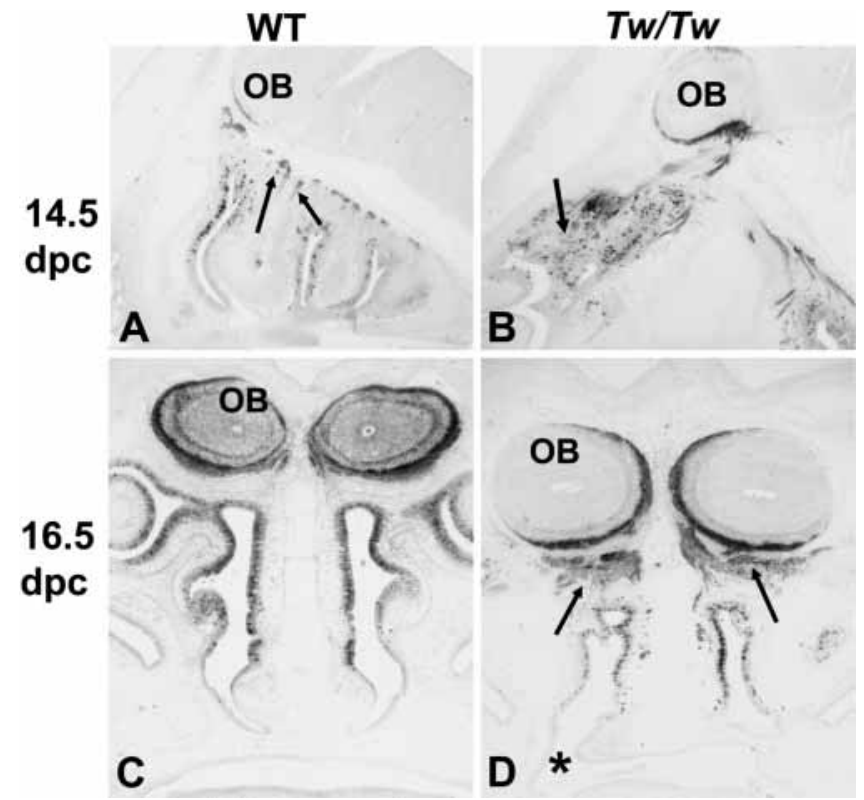

Fig. 4 Distribution of olfactory nerves in 14.5 and 16.5 dpc embryos. The distribution of olfactory nerves as marked by their positive immunoreactivity with the antibody against omp in $14.5 \mathrm{dpc}$ embryos (top panels; sagittal sections) and 16.5 embryos (bottom panels; coronal sections) of wildtype (left panels) and Tw/Tw (right panels). In wildtype embryos, axons from ORN in the olfactory epithelium aggregate into bundles to penetrate foramina in the cribriform plate (arrows in A) to form a layer in the outermost part of the $\mathrm{OB}(\mathbf{A}, \mathbf{C})$. In the $T w / T w$ embryo, olfactory nerves from the olfactory epithelium appear randomly scattered in the mesenchyme of the nasal cavity (arrow in B). In these embryos, some fasciculi reached the $\mathrm{OB}$ on its underside $(\mathbf{B}, \mathbf{D})$ and medial surface (D). Some of the bundles accumulated in the mesenchyme just below the OB (D). The secondary palate in the $T w$ homozygote is clefted (asterisk in D).

rows in Fig. 4D). As the cribriform plate was frequently disrupted in these animals, the olfactory nerves did not appear to penetrate any obvious bony structures or foramina in the cribriform plates. In addition to the random distribution in the nasal region, very few of these nerves appeared to reach the OB. This resulted in a reduced layer of olfactory nerve in the $\mathrm{OB}$ in the newborns compared to that of the wildtype (compare Fig. 3C and 3D). The different layers of the OB that were distinct in the wildtype embryos were not apparent in the $T w / T w$ embryos. Not only were the glomerular layer virtually nonexistent (Fig. 3D), the mitral cells appeared to be scattered over the entire surface of the OB (Fig. 3F). As the olfactory axons terminate within a glomerulus, where they synapse upon mitral, juxtaglomerular, and periglomerular neurons, it is assumed that fewer synapses were formed in these mutant mice.

\section{Olfactory epithelium}

To analyze the development of the olfactory receptor neurons (ORN), we performed histological analysis with 
hematoxylin and eosin, immunostaining with the PGP 9.5 and omp antibodies on embryos at 14.5 and 16.5 days of development and on newborn pups. By day 14 of development, it was possible to detect differentiation and layering of nuclei within the olfactory epithelium, a simple neuroepithelium comprising cell populations distinguishable by their morphology and laminar position (basal cells, olfactory neurons, and sustentacular cells; Graziadei and Monti-Graziadei, 1978, 1979). This stratification of about 8-9 layers thick was already evident in the olfactory epithelium of wildtype embryos at 14.5 days of development (Fig. 5A). There was a distinction between the elongate, heterochromatic nuclei at the apex and the more rounded and euchromatic ones situated more deeply, all of which were arranged in columns perpendicular to the epithelial surface with no sharply defined boundaries. Cells in the middle layer of cells contained ORN at different stages of maturation and stained positive for the PGP 9.5 antibody (Fig. 5C).

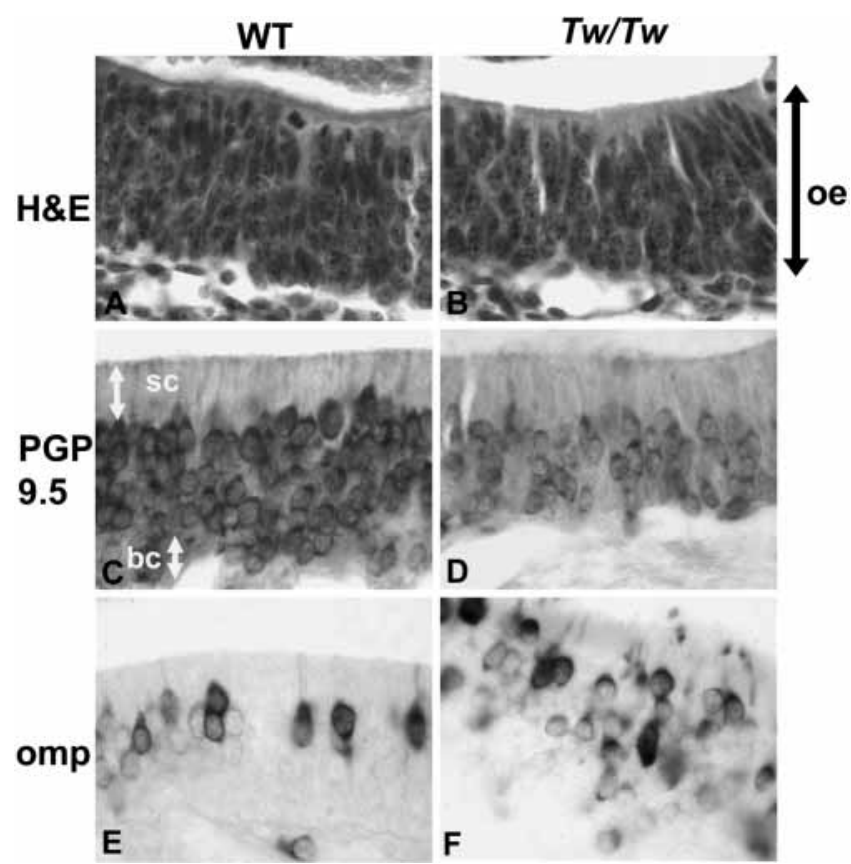

Fig. 5 Phenotype of olfactory epithelium in 14.5 dpc mice. Sagittal sections through the olfactory epithelium (oe) of $14.5 \mathrm{dpc}$ wildtype (left panels) and $T w / T w$ embryos (right panels). The nasal cavity is toward the top and the lamina propria, the bottom. $\mathbf{E}$ and $\mathbf{F}$ are at higher magnification $(50 \times)$. A-B Hematoxylin and eosin $(H \&$ $E)$ stained sections. A Epithelial supporting or sustentacular cells (sc) form one layer on the apical side of wildtype mature olfactory epithelium. The basal cells $(b c)$ are the progenitors of the olfactory neuron lineage and are found on the basal side of the epithelium. Olfactory receptor neurons (ORN) are found between these two layers as evidenced by their immunoreactivity to the PGP 9.5 antibody ( $\mathbf{C}$ and $\mathbf{D})$. Contained amongst these cells are some that are fully differentiated with their dendrites and are therefore $\mathrm{omp}^{+}(\mathbf{E})$. In contrast, in the wildtype olfactory epithelium, the layer of the epithelium is slightly thinner, about 6 layers thick. The layer of ORN is thinner (D) and contained amongst them are mature ORN with dendrites scattered randomly in the epithelium.
E14.5 is the first stage at which mature ORN were first observed in large numbers in wildtype embryos (Farbman and Margolis, 1980). Maturation of the olfactory neuroepithelium is paralleled by a progressive increase in the number of neurons containing the olfactory marker protein (Margolis, 1972, 1988) and olfactory cilia (Chuah et al., 1985). Therefore, within this middle layer of cells, a few of the ORN were mature as evidenced by their reactivity to the omp antibody. When the olfactory epithelium of $14.5 \mathrm{dpc} T w / T w$ embryos was compared to that of their wildtype littermates, the olfactory epithelium appeared slightly thinner (about 6 layers thick compared to the 8-9 layers found in wildtype; Fig. 5B and D). Mature ORN with their cilia appeared numerous in the homozygotes; these ORN were scattered throughout the epithelium, unlike those in the wildtype, with a few ORN present in the mesenchyme (Figs 4B and 5D).

By $16.5 \mathrm{dpc}$ in wildtype embryos, the rounded nuclei of the developing receptors contrasted with the more elongate forms of the embryonic stem cells (Fig. 6A, C, E). The middle layer of the epithelium in wildtype embryos had become more mature and differentiated compared with an earlier stage $(14.5 \mathrm{dpc})$, with the cells in

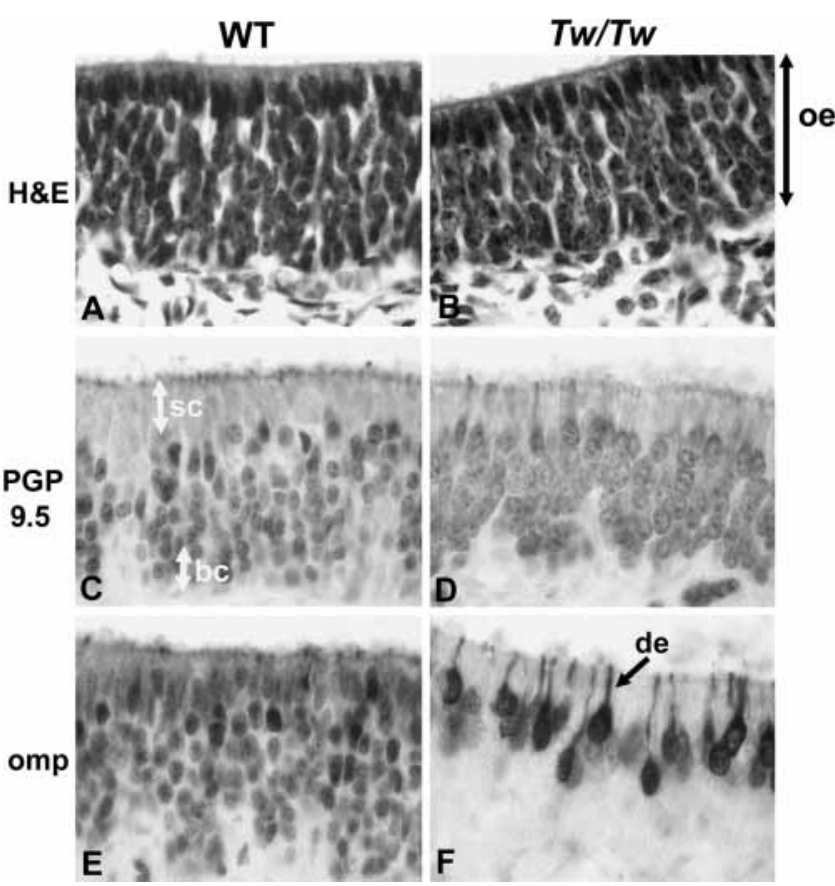

Fig. 6 Phenotype of olfactory epithelium in 16.5 dpc mice. The sections are oriented and organized the same way as in Fig. 5. In the H\&E-stained sections, the difference between the wildtype (A) and $T w / T w$ is visible with the ORN maintaining the bulbous shape. In wildtype embryos, the ORN are slightly rounded $(\mathbf{A}, \mathbf{C})$, becoming more mature compared to an earlier age, less rounded. ORN did not appear to have undergone the differentiation process and are short and bulbous. Some of these ORN are immunoreactive with the omp antibody. oe = olfactory epithelium; sc = sustentacular cells; $b c=$ basal cells; de $=$ dendrites. 


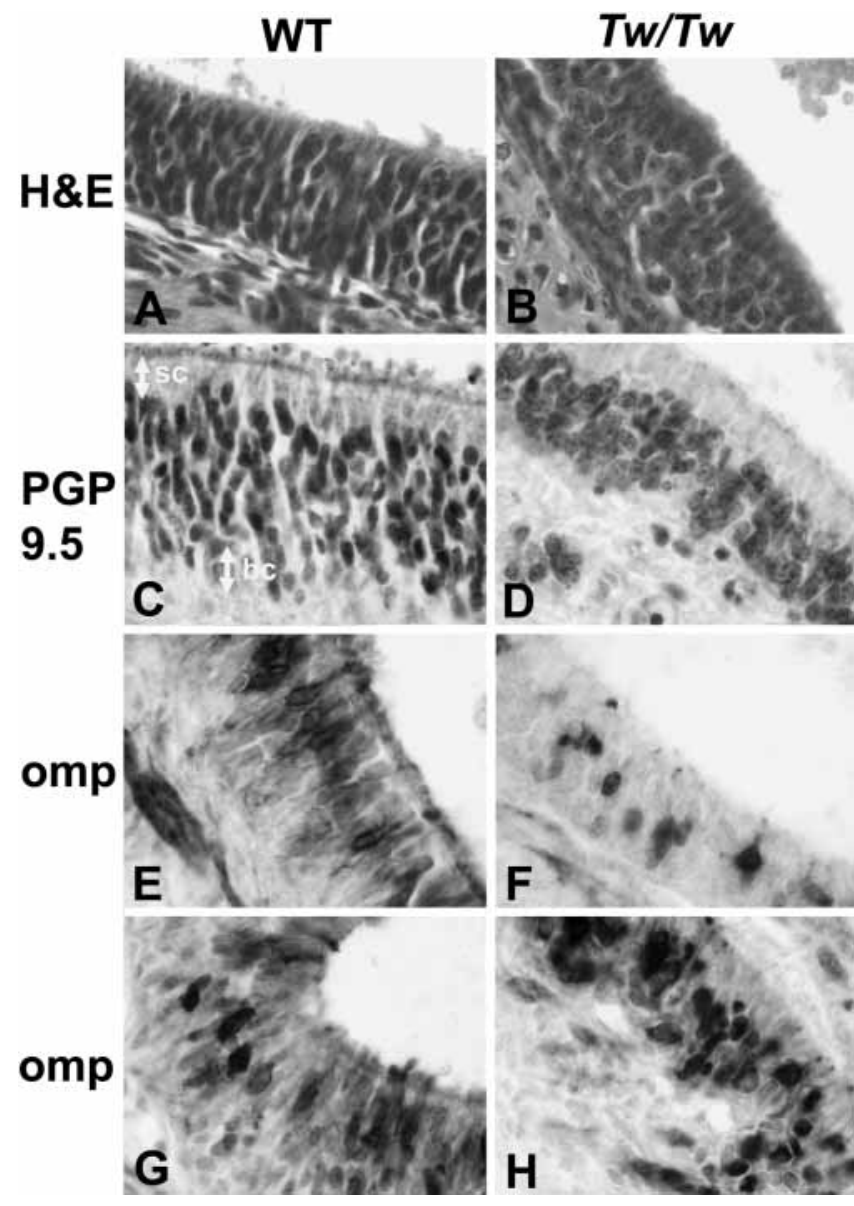

Fig. 7 Phenotype of olfactory epithelium in newborn mice. The sections are oriented the same way as in Fig. 5. At birth, the olfactory epithelium basically retained the same kind of pattern that was observed in $16.5 \mathrm{dpc}$ embryos. The olfactory epithelium was thinner in the $T w$ homozygotes with the ORN less differentiated than those of wildtype. The distribution of $\mathrm{omp}^{+}$was scattered unevenly throughout the epithelium with some parts having more (H) and other parts less $(\mathbf{F}) . \mathrm{sc}=$ sustentacular cells; $\mathrm{bc}=$ basal cells.

this layer becoming more heterochromatic, less rounded, and immunoreactive with the omp antibody (Fig. 6A, C, E). In the $T w / T w$ embryos, the ORN did not appear to have undergone the differentiation process of becoming elongated, maintaining the rounded shape and euchromatism of ORN present in younger embryos (Fig. 6B, $\mathrm{D}, \mathrm{F})$. Some ORN in the olfactory epithelium of $T w$ homozygotes were $\mathrm{omp}^{+}$, exhibiting strong immunoreactivity with the antibody especially in the perikarya continuing into dendrites that reached the epithelial surface; these ORN, when compared to the ORN in their wildtype littermates, were much fewer and bigger in size (Fig. 6F). At birth, the olfactory epithelium basically retained the same kind of pattern that was observed in $16.5 \mathrm{dpc}$ embryos. The olfactory epithelium was thinner in the $T w$ homozygotes with the ORN less differentiated than those of wildtype. The distribution of $\mathrm{omp}^{+}$was scattered unevenly throughout the epithelium with some parts having more (Fig. $7 \mathrm{H}$ ) and other parts less (Fig. $7 \mathrm{~F})$.

\section{Discussion}

The olfactory epithelium undergoes constant regeneration, with self-renewal from a stem cell that gives rise to new ORN. We have used both the simple layered organization and the immunohistochemical reactivity to two well-characterized antibodies of the olfactory epithelium to facilitate the histological identification and to assess their different stages of development. We chose to analyze embryos starting at 14.5 days of development due to the fact that the first synapses between sensory axons and mitral cell dendrites in the $\mathrm{OB}$ are seen on the 14th embryonic day.

The disruption in the OB appeared to be much more visible at birth, with severe alterations in their shape and size. This was in contrast to the younger staged embryos where the $\mathrm{OB}$ appeared relatively normal in size and shape. Similarly, the defects in the skeletal structures of the cranial base under the OB were more severe in newborn pups compared to embryos. This begs the question of whether the defects in the skeletal structures were present prior to the development of the $\mathrm{OB}$, leading to the abnormal development of the $\mathrm{OB}$, or whether it was due to the abnormal development of the OB that prevented normal development of the bony components. We suspected that it was due to the latter because of the fact that abnormal development of the ethmoid bone was not observed in younger embryos. One could speculate that the OB developed abnormally in $T w$ homozygotes due to missing intrinsic cues resulting from the $T w$ gene mutation; the abnormally shaped olfactory bulb subsequently caused the surrounding skeletal structures to be abnormally formed.

The olfactory innervation was altered in the Twirler mice with a reduced number of connections in the olfactory bulb. In mutant embryos at 14.5 days, many mature ORN, as evidenced by their positive immunoreactivity to the omp antibody, appeared to be found randomly all over the mesenchyme compared to those in the wildtype littermate. Since the first synapses are in the process of being formed during this period, it is likely that many of these axons had problems reaching their target, the $\mathrm{OB}$, at this early stage. The lack of guidance of the axons in migrating to the OB could be due to a lack of guidance molecules. In this regard, it is interesting to note that semaphorins and their receptors have been identified as putative molecular cues involved in olfactory pathfinding (Pasterkamp et al., 1999; Williams-Hogarth et al., 2000). Most studies have indicated a chemorepulsive role for the semaphorins for axon pathfinding, branching, or targeting (Mark et al., 1997). It is possible that in the $T w$ homozygous mice, some semaphorins 
and/or other molecules may not be providing the right signals for the axons in their migratory pathways to the OB. Until the localization and expression of members of the semaphorin family and/or their receptors has been performed in the craniofacial and central nervous system of $T w / T w$ mice, the role of these genes in olfactory pathogenesis in $T w$ mice remains purely speculative.

By $16.5 \mathrm{dpc}$, fasciculation of the axons in the mesenchyme in both homozygotes and wildtype mice was more obvious, although in the homozygotes, the pattern was not in as orderly a manner as those in the wildtype littermates. Again, the number of axons reaching the outer layer of the OB was much reduced in most places in the $T w$ homozygotes and the axons did not appear to make synapses with the mitral cells as evidenced by a lack of glomerular layer in the OB. It thus appears that the ORN in the $T w$ homozygotes did not make contact with the OB. In addition, the ORN in the $T w$ homozygotes developed initially, but differentiation of these neurons did not keep pace with those of the wildtype littermates. This was in contrast to mice in whom the Mashl gene was disrupted where the numbers of ORN generated during development were greatly reduced (Guillemot et al., 1993, 1995). In the $T w / T w$ mice, the number of layers of cells in the olfactory epithelium, although thinner, was still evident, but the ORN were immature and undifferentiated. The disruptions in the OB and the ORN in the olfactory epithelium appear to be interconnected. In the olfactory system, there are developmental interactions between the sensory neurons in the nasal cavity and their target, the OB (Farbman, 1988). The final maturation of the ORN in the epithelium depends on proper influence of the $\mathrm{OB}$ as it has been shown that formation of cilia, where the receptive apparatus of the ORN is located, was completed only after the axons reached the bulb (Cuschieri and Bannister, 1975). In the chronic absence of the bulb, the epithelium remains thinner than normal with a marked reduction in the number of ORN that express the omp, indicating that average neuronal life span was reduced in the ablated side, and that, therefore, the ORN are trophically dependent on the presence of the bulb for their prolonged survival (Monti-Graziadei, 1983; Verhaagen et al., 1990; Schwob et al., 1992). The olfactory phenotype of the $T w$ mice appeared to represent partial bulbectomy where stratification of the olfactory epithelium was still present, albeit less distinct, and fewer of the ORN were mature and differentiated. The fact that the axons at an early age $(14.5 \mathrm{dpc})$ were remiss in getting to their target would be similar to a partial bulbectomy.

It is expected that the olfactory defects in the $T w / T w$ would lead to some kind of olfactory function deficits. Assays of olfactory function unfortunately cannot be performed on the $T w$ homozygotes as they die soon after birth. It is thus not possible to ascertain directly whether the observed differences in distribution of omp and PGP 9.5 in the axons and cell bodies of ORN and changes in cell morphology of the olfactory epithelium of $T w / T w$ are secondary to the craniofacial malformations associated with the $T w$ mutation. Perhaps more sophisticated physiological assays can be used on cultures of olfactory harvested from $T w / T w$ embryos to test for olfactory function tissues (use of olfactory cultures reviewed in Calof et al., 1995).

Are the defects present in other parts of the midface related to the olfactory defects in the $T w$ mice? The primordium of the olfactory organ is the olfactory placode, from which many structures of the midface, including the primary and secondary palates, develop. All $T w$ homozygotes also present with clefts of the lip and palate. $T w$ heterozygotes and homozygotes, in addition, have defects in inner ear development (Lyon, 1958; personal observations). The defects in these mice represent alterations in the morphology of the midface, e.g. clefts of the lip and palate, and the shape of the inner ear, e.g. abnormally shaped semicircular canals and cochlea. The defects all appeared to arise from a lack of signals regulating the changes during development such as fusion of the facial processes during midfacial morphogenesis. The cloning of the $T w$ gene will help to reveal the mechanism(s) that operate during the development of the midface, the olfactory circuitry, and the inner ear. In this light, it is interesting to note the existence of some human conditions that resemble to some extent the phenotype observed in $T w / T w$ mice, e.g. two male humans have been identified that had hypoplasia of the nose and eyes, together with hyposmia, hypogeusia, and hypogonadism (Bosma et al., 1981).

The identification of a number of factors and molecules that regulate proliferation, differentiation, and renewal of ORN and their precursors suggest that there are multiple developmental stages that may serve as control points for the proper generation of the right cell number, maturation, and axonal migration and guidance of the ORN. The Twirler mice can provide another model system to evaluate the developmental events that lead to maturation and pathfinding of these ORN.

Acknowledgements We thank Dr. Frank Margolis for his generous gift of antiserum to olfactory marker protein. The technical assistance of Susan Rynkiewicz and Chiao Guo and intellectual input from Dr. G. R. Holland are greatly appreciated. This study was supported by funds from the National Institute of Dental and Craniofacial Research (RO3DE12048-01), Cleft Palate Foundation, and the American Association of Orthodontist Foundation.

\section{References}

Bosma, J.F., Henkin, R.I., Christiansen, R.L. and Herdt, J.R. (1981) Hypoplasia of the nose and eyes, hyposmia, hypogeusia, and hypogonadotrophic hypogonadism in two males. J Craniofac Genet Dev Biol 1:153-184.

Buck, L.B. (1996) Information coding in the vertebrate olfactory system. Annu Rev Neurosci 19:517-544.

Caggiano, M., Kauer, J.S. and Hunter, D.D. (1994) Globose basal 
cells are neuronal progenitors in the olfactory epithelium: A lineage analysis using a replication-incompetent retrovirus. Neuron 13:339-352.

Calof, A.L., Hagiwara, N., Holcomb, J.D., Mumm, J.S. and Shou, J. (1996) Neurogenesis and cell death in olfactory epithelium. J Neurobiol 30 (1):67-81.

Chuah, M.I., Farbman, A.I. and Menco, B.P.M. (1985) Influence of olfactory bulb on dendritic knob density of rat olfactory receptor neurons in vitro. Brain Res 338:259-266.

Cuschieri, A. and Bannister, L.H. (1975) The development of the olfactory mucosa in the mouse: light microscopy. J Anat 119 (2):277-286.

Farbman, A.I. (1988) Cellular interactions in the development of the vertebrate olfactory system. In: Margolis, F.L. and Getchell, T.V. (eds) Molecular neurobiology of the olfactory system. Plenum Press, New York, pp 319.

Farbman, A.I. (1991) Developmental neurobiology of the olfactory system. In: Getchell, T.V., Doty, R.L., Bartoshuk, L.M. and Snow Jr., J.B. (eds) Smell and Taste in Health and Diseases. Raven Press, New York, pp 3333-4444.

Farbman, A.I. and Margolis, F.L. (1980) Olfactory marker protein during ontogeny: immunohistochemical localization. Dev Biol 74:205-215.

Gong, S.-G. and Eulenberg, R. Palatal development in Twirler mice. Cleft Palate Craniofac J. In press.

Gong, S.-G., White, N.J. and Sakasegawa, A.Y. (2000) Twirler: A mouse model to study clefts of the lip and palate. Arch Oral Biol 45 (1):87-94.

Graziadei, P.P. (1973) Cell dynamics in the olfactory mucosa. Tissue Cell 5 (1):113-131.

Graziadei, P.P. and Metcalf, J.F. (1971) Autoradiographic and ultrastructural observations on the frog's olfactory mucosa. Z Zellforsch Mikrosk Anat 116 (3):305-318.

Graziadei, P.P.C. and Monti-Graziadei, G.A. (1978) Continuous nerve cell renewal in the olfactory system. In: Jacobson, M. (ed) Handbook of sensory physiology, Vol IX. Development of sensory systems. Springer, Berlin, pp 55-83.

Graziadei, P.P.C. and Monti-Graziadei, G.A. (1979) Neurogenesis and neuron regeneration in the olfactory system of mammals. I. Morphological aspects of differentiation and structural organization of the olfactory sensory neurons. J Neurocytol 8:1-18.

Guillemot, F., Lo, L.C., Johnson, J.E., Auerbach, A., Anderson, D.J. and Joyner, A.L. (1993) Mammalian achaete-scute homolog 1 is required for the early development of olfactory and autonomic neurons. Cell 5:463-476.

Guillemott, T. (1995) Analysis of the role of basic helix-loop-helix transcription factors in the development of neural lineages in the mouse. Biol Cell 84:3-6.

Jones, N. and Rog, D. (1998) Olfaction: a review. J Laryngol Otol 112:11-24.

Kinnamon, S. and Getchell, T. (1991) Sensory transduction in olfactory receptor neurons and gustatory receptor cells. In: Getchell, T.V., Doty, R.L., Bartoshuk, L.M. and Snow Jr., J.B. (eds) Smell and Taste in Health and Disease Raven Press, New York, pp 145-172.
Lyon, M.F. (1958) Twirler: A mutant affecting the inner ear of the house mouse. J Embry Exp Morph 6:105-116.

Margolis, F.L. (1972) A brain protein unique to the olfactory bulb. PNAS USA 69:1221-1224.

Margolis, F.L. (1975) Biochemical markers of the primary olfactory pathway: a model neural system. In: Agranoff, B.W. and Aprison, M.H. (eds) Advances in Neurochemistry. Plenum Press, New York, pp 193-246.

Margolis, F.L. (1988) Molecular cloning of olfactory specific gene products. In: Margolis, F.L. and Getchell, T.V. (eds) Molecular Neurobiology of the Olfactory System. Plenum Press, New York, pp 237-265.

Mark, M., Cohrun, M. and Pushchel, A. (1997) Patterning neural connections by chemorepulsion: the semaphorins. Cell Tissue Res 290:299-306.

Monti-Graziadei, G.A. (1983) Experimental studies on the olfactory marker protein. III. The olfactory marker protein in the olfactory neuroepithelium lacking connections with the forebrain. Brain 262:303-308.

Monti-Graziadei, G.A., Margolis, F.L., Harding, J.W. and Graziadei, P.P.C. (1977) Immunocytochemistry of the olfactory marker protein. J Histochem Cytochem 25:1311-1316.

Moulton, D.G. (1974) Dynamics of cell populations in the olfactory epithelium. Ann NY Acad Sci 237:52-61.

Moulton, D.G., Celebi, G. and Fink, R.P. (1970) Olfaction in mammals-Two aspects: proliferation of cells in the olfactory epithelium and sensitivity to odours. In: Wolstenholme, G.E.W. and Knight, J. (eds) Taste and smell in vertebrates. Churchill, London, pp 241-252.

Pasterkamp, R.J., Ruitenberg, M.J. and Verhaagen, J. (1999) Semaphorins and their receptors in olfactory axon guidance. Cell Mol Biol 45 (6):763-779.

Schwob, J.E., Mieleszko, S. and Stasky, A.A. (1992) Olfactory sensory neurons are trophically dependent on the olfactory bulb for their prolonged survival. J Neuroscience 12 (10):3896-3919.

Shepherd, G.M. (1994) Discrimination of molecular signals by the olfactory receptor neuron. Neuron 13:771-790.

Thompson, R.J., Doran, J.F., Jackson, P., Dhillon, A.P. and Rode, J. (1983) PGP9.5-a new marker for vertebrate neurons and neuroendocrine cells. Brain Res 278 (1-2):224-228.

Verhaagen, J., Oestreicher, A.B., Grillo, M., Khew-Goodall, Y.-S., Gispen, W.H. and Margolis, F.L. (1990) Neuroplasticity in the olfactory system: differential effects of central and peripheral lesions of the primary olfactory pathway on the expression of B50/GAP43 and the olfactory marker protein. J Neurosci Res 26:31-44

Williams-Hogarth, L.C., Puche, A.C., Torrey, C., Cai, X., Song, I., Kolodkin, A.L., Shipley, M.T. and Ronnett, G.V. (2000) Expression of semaphorins in developing and regenerating olfactory epithelium. J Comp Neurol 423:565-578.

Wilson, P.O., Barber, P.C., Hamid, Q.A., Power, B.F., Dhillon, A.P., Rode, J., Day, I.N., Thompson, R.J. and Polak, J.M. (1988) The immunolocalization of protein gene product 9.5 using rabbit polyclonal and mouse monoclonal antibodies. Br J Exp Path 69 (1):91-104 